\title{
Eine humanitäre und politische Reise nach Rojava
}

\section{Maja Hess ${ }^{a}$, Bigna Rambert ${ }^{b}$}

${ }^{a}$ med. pract., Präsidentin von medico international schweiz; ${ }^{b}$ Dr. med., FMH für Psychiatrie und Psychotherapie

Der Reisebericht von der türkisch-syrischen Grenze schildert den unermüdlichen Einsatz kurdischer Ärztinnen und Freiwilliger mitten im Krieg. Er zeigt auf, dass in einer globalisierten Welt im Mittleren Osten auch ein Stellvertreter-Krieg geführt wird. Ein Krieg gegen faschistoide, religiös entartete und rassistische Gewalt, ein Kampf für emanzipatorische und demokratische Ideen auf der ganzen Welt.

Roj ist kurdisch, wird Rosch ausgesprochen, heisst Sonne, aber auch Tag. Rojava könnte wörtlich als Land des Sonnenuntergangs übersetzt werden. Im Schatten des syrischen Bürgerkrieges riefen kurdische Organisationen vor einem Jahr zum Autonomie-Projekt Rojava auf. Es umfasst drei nicht miteinander verbundene Kantone im Norden und Nordosten Syriens: Efrin, Kobanê und Cîzîre.

Rojava ist ein ambitiöses, ziviles, regionales Projekt: Demokratischer Konföderalismus, Rätestrukturen, kooperative Selbstverwaltung, Geschlechtergerech- tigkeit, Säkularismus, Multireligiosität und Multiethnizität werden in Rojava mitten im Krieg von vielen engagierten Menschen erprobt. Auffällig viele Frauen sind an diesem Projekt beteiligt.

Die Stadt Kobanê, im kleinsten der drei Kantone, sorgte in der Weltpresse wegen der Belagerung durch den «Islamischen Staat» (IS) lange für Schlagzeilen. Unsere fünfköpfige Gruppe fährt in die türkische Grenzstadt Suruç, die Nachbarstadt von Kobanê.

Wir sind drei Ärztinnen, eine Körpertherapeutin mit Arabischkenntnissen und unsere Fahrerin. Wir reisen

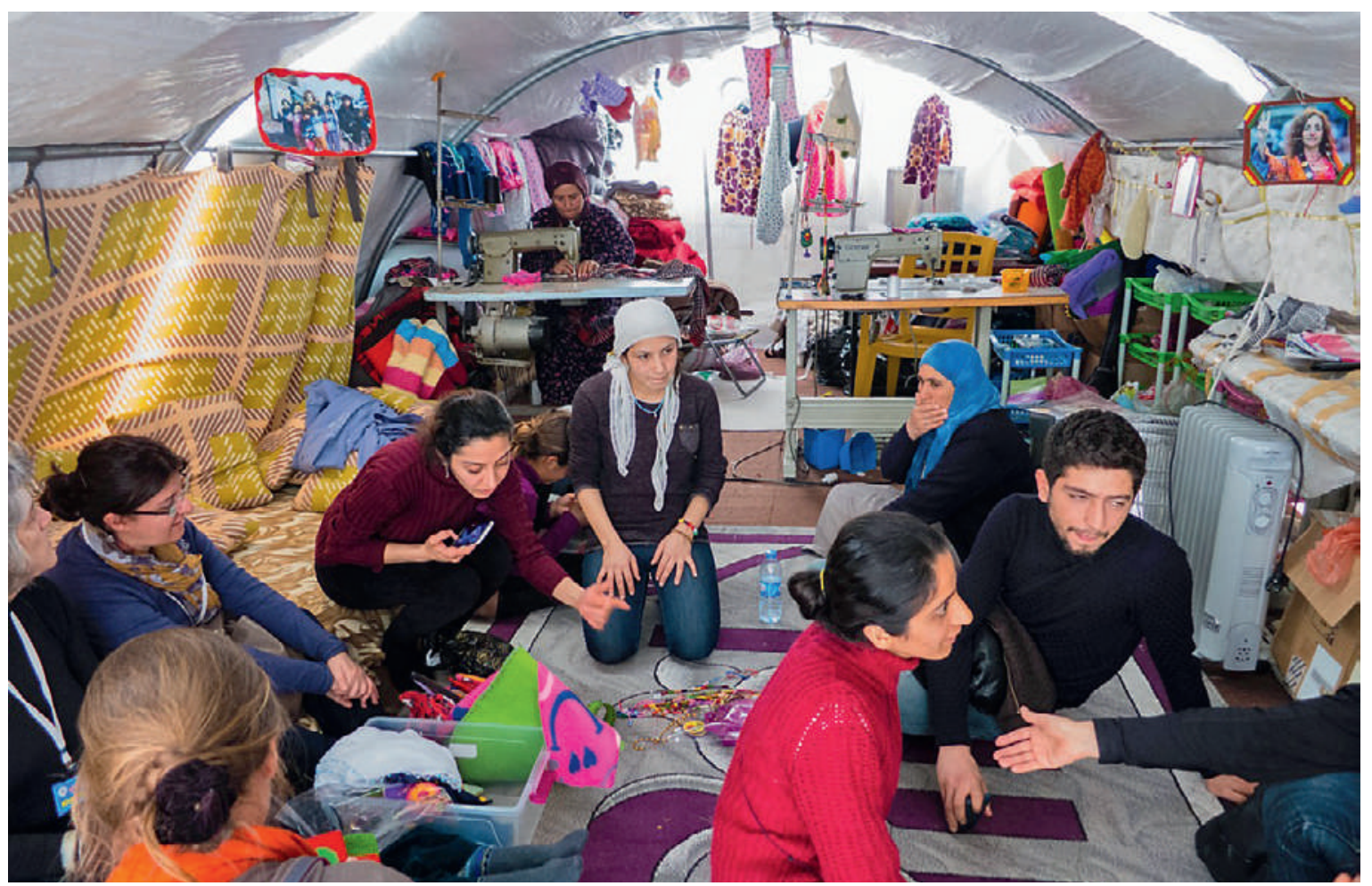

Endlich etwas Freude im Nähatelier des Flüchtlingslagers von Suruç an der türkisch-syrischen Grenze: In Kobanê wurde ein Sieg über den IS erkämpft. 


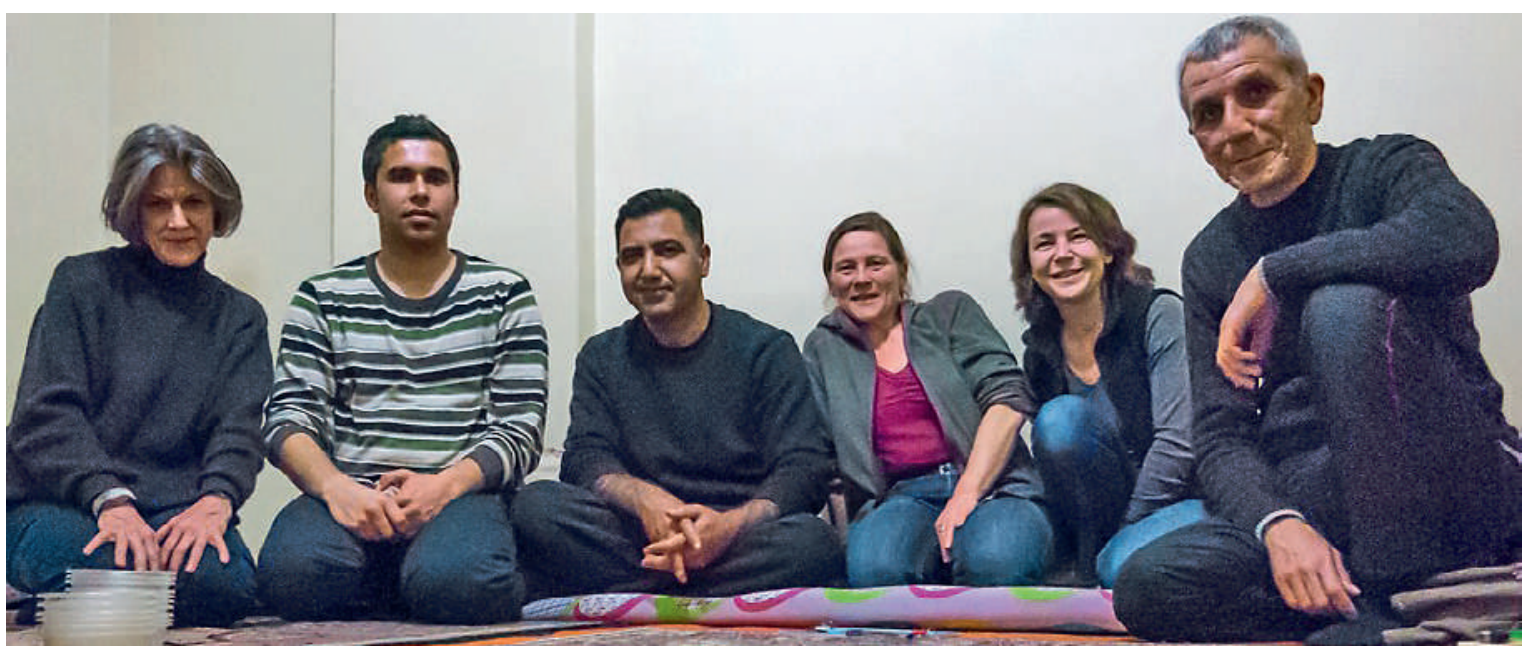

Lächeln trotz lebensbedrohlicher Arbeitsbedingungen inmitten grossen Leids: Ärzte aus Kobanê.

mit einem Abklärungsauftrag für medico international Schweiz ( ehemals Centrale Sanitaire Suisse) einer kleinen NGO, die während des spanischen Bürgerkriegs gegründet wurde.

Die knapp dreistündige Fahrt von Diyarbakir nach Suruç führt durch karge anatolische Steinlandschaften mit vereinzelten Schafherden. Am Weg liegt Urfa - eine historische muslimische Pilgerstadt. Nach einem Empfang im Krisen- und Koordinationszentrum Rojava in Suruç besuchen wir das Dorf Meresat. Nur wenige Kilometer entfernt, auf der anderen Seite der Grenze, liegt die Stadt Kobanê. Sieben Tage vor unserer Ankunft wurde sie durch kurdische Kämpfer und Kämpferinnen zurückerobert und vom IS befreit.

\section{Freude trotz anhaltender Bedrohung}

In Meresat herrscht Dunkelheit, ein lautloses Kommen und Gehen und Freude über den Sieg über den IS: An einer Steinwand hängen verschiedene selbstgebastelte, kämpferische Plakate, in einem Zelt woh-

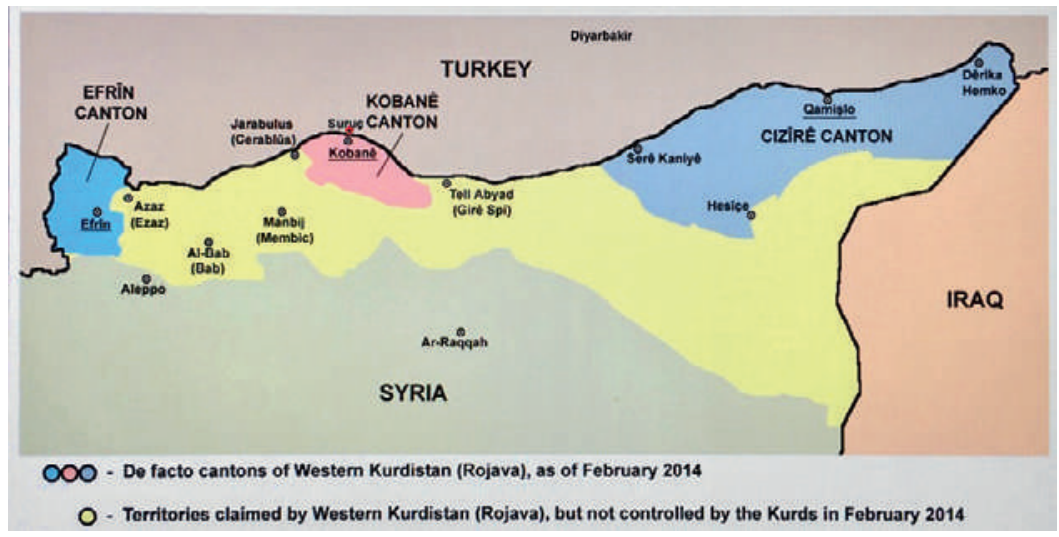

Das Grenzgebiet zwischen der Türkei und Syrien. Das Krisen- und Koordinationszentrum Rojava befindet sich in Suruç. nen Menschenrechtsaktivisten aus Diyarbakir, um ein Feuer stehen Menschen und erwärmen sich. Wir lernen, dass das kurdische Wort azadî Freiheit heisst. Eine Frau macht uns ein Victory-Zeichen. Wie viele andere Mütter kam sie seit Beginn des Krieges von weit her immer wieder in dieses Dorf. Ihre Tochter

\section{Alle drei Spitäler der Stadt Kobanê aus der} Vorkriegszeit wurden im Laufe der Belagerung zerstört.

kämpft in Kobanê. Die Lage ist immer noch gefährlich. Die um Kobanê liegenden Dörfer müssen noch vom IS befreit werden. Wir schauen dem Blick dieser Mutter folgend in die schwarze Dunkelheit.

\section{Erschütternde Erzählungen der Ärzte}

Die uns vom Rojava Krisen- und Koordinationszentrum zugewiesene Unterkunft ist zugleich die bescheidene Dienstwohnung dreier Ärzte aus Kobanê. Ihre Erzählungen sind erschütternd: Alle drei Spitäler der Stadt Kobanê aus der Vorkriegszeit wurden im Laufe der Belagerung zerstört. Ebenso die Blutbank von medico international Deutschland. Mit Hilfe kurdischer Chirurgen aus Diyarbakir versuchten auch diese drei Kollegen, unter prekärsten Bedingungen während der Belagerung eine medizinische Erstversorgung aufrechtzuerhalten. Nur durch einen Zufall überlebten sie im letzten Kriegsmonat ein Selbstmordattentat auf das letzte noch einigermassen funktionierende Spital.

Auch jetzt noch fahren sie täglich im Turnus über die Grenze, um in einer Art Kellerspital zu arbeiten. 80\% der Stadt sind zerstört. Aktuell drohen vor allem Verletzungen durch weitere Einstürze von Häusern, durch Blindgänger und durch von dem IS zurückge- 


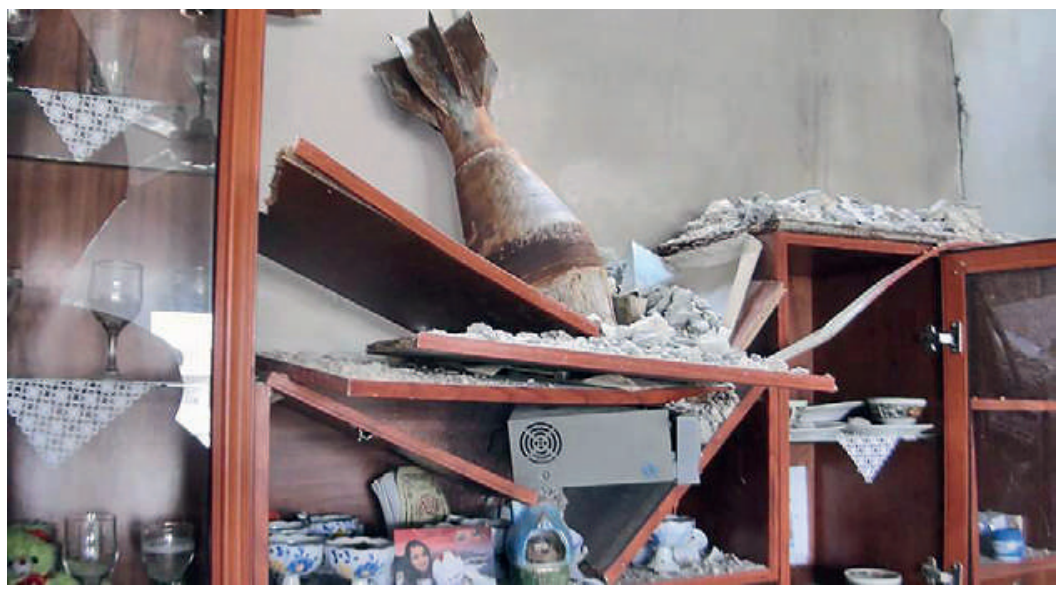

Alltag in Kobanê.
Wir besuchen vier Lager. Der Sieg über den IS spiegelt sich in den Gesichtern der Menschen und in ihren Erzählungen wider. Das Wetter ist mild und die unzähligen Kinder spielen fröhlich um die Zelte. Die Stimmung ist hoffnungsvoll und berührend.

Struktur und Sicherheit sowie die Abgabe von Essen und die medizinische Versorgung werden durch das Rojava Krisen- und Koordinationszentrum gewährleistet. In jedem Lager gibt es einen Sanitätsdienst. Die ärztliche Versorgung wird in freiwilliger Arbeit von kurdischen Ärztinnen aus verschiedenen Städten aus dem Südosten der Türkei im Turnus durchgeführt. Die kurdische Gesundheitsgewerkschaft ist federführend. Eine gewaltige Aufgabe auch für die organisationserprobten Kurden.

lassene Sprengfallen. Kurz vor unserer Rückreise nach Diyarbakir besuchen wir im Spital in Suruç den Spitalverwalter aus Kobanê. Er war dort durch eine herunterstürzende Decke schwer verletzt worden. Ein weiteres grosses Problem sind die vielen toten ISKämpfer, die noch unter den Trümmern vor allem im Osten der Stadt liegen.

"Wissen die Menschen in Europa eigentlich, dass wir hier in Kobanê gegen den IS auch für Europa einen Stellvertreter-Krieg führen? Nach dem Angriff auf ¿Charlie Hebdo» sollte ihnen das doch klar sein?», fragt uns ein 33-jähriger Internist. Auf seinem Handy zeigt er uns Fotos seiner Heimat, ein friedliches Bauerndorf, das heute im IS-Gebiet im Nordosten Syriens liegt. Er ist syrischer Kurde, spricht arabisch, hat einen arabischen Vornamen und studierte in Aleppo Medizin. Unter dem al-AssadRegime war Kurdisch in Syrien ebenso wie in der Türkei Erdogans verboten. Heute würde er lieber einen kurdischen Namen tragen, würde lieber Reber (deutsch: Wächter) heissen. Eine Schwester und ein Bruder und weitere nahe Verwandte starben in den Reihen der kurdischen Volksverteidigungskämpferinnen und kämpfer. Ebenso wie viele andere Flüchtlinge, die wir später in den Lagern treffen, will er so bald wie möglich wieder in Kobanê leben. Über ihn stossen wir auf den Artikel Les Kurdes nous défendent tous des ermordeten Stéphane Charbonnier, Herausgeber von Charlie Hebdo. Er hatte diesen kurzen Aufruf, drei Monate vor seinem Tod, geschrieben (L'Humanité, 22.10.14).

\section{Die Organisation der Flüchtlingslager}

Das Rojava Krisen- und Koordinationszentrum in Suruç betreut mit Hilfe der lokalen kurdischen Stadtregierung sechs Flüchtlingslager mit ca. 13800 Flüchtlingen aus Kobanê und den umliegenden Dörfern.
Das türkische Katastrophenhilfswerk Afad hat in Suruç ebenfalls ein Lager eingerichtet. Die ca. 7000 Zelte seien nur zur Hälfte belegt. Zu gross sei das Misstrauen vieler Kurdinnen und Kurden gegenüber dem türkischen Staat angesichts der zynischen Politik der Regierung Erdogan. Kämpfer des IS würden über die Grenze nach Syrien eingeschleust, die Ausreise kurdischer Kämpferinnen und Kämpfer behindert. Der Grenzverkehr zwischen Suruç und Kobanê ist von der türkischen Regierung grundsätzlich geschlossen.

\section{Wir waren hin- und hergerissen zwischen} unserer europäischen Skepsis und ihrem Mut und ihrer Unerschrockenheit mitten im Krieg.

Möglicherweise hätte das Leben einiger Kämpfer gerettet werden können. Ambulanzen mit Verletzten aus Kobanê seien an der türkischen Grenze unnötig behindert worden.

Am zweiten Tag unseres Besuches in Suruç findet eine Pressekonferenz statt. Die lokale kurdische Regierung und das Rojava Krisen- und Koordinationszentrum fordern von der türkischen Regierung endlich einen offenen Korridor zwischen Suruç und Kobanê. Bis heute ist er nicht eingerichtet.

Mit dem Rojavaprojekt identifizieren sich verschiedenste fortschrittliche kurdische Parteien, SammelBewegungen und Einzelpersonen in der ganzen Region. Die Geschichte innerkurdischer Differenzen, Allianzen, Parteigründungen und Abspaltungen in der Türkei, aber auch in Syrien ist lang und war auch immer wieder blutig. Sowohl türkische als auch syri-

"Gegen die IS reichen Worte nicht mehr aus», so beginnt de Aufruf von medico international schweiz für ein Nothilfeprojekt zur medizinischen Unterstützung der Flüchtlinge und der kurdischen Kämpfenden aus Kobanê: Spendenkonto 80-7869-1, Vermerk Kobanê. 
sche Regimes haben im letzten Jahrhundert diese Rivalitäten unter den Kurden für ihre Herrschaftssicherung jeweils brutal ausgenützt.

Sowohl in Suruç als auch in Diyarbakir hatten wir immer wieder Gelegenheit, dem unermüdlichen politischen Veränderungs- und Gestaltungswillen unserer Gastgeberinnen und Gastgeber zu begegnen. Wir waren hin- und hergerissen zwischen unserer europäischen Skepsis und ihrem Mut und ihrer Unerschrockenheit mitten im Krieg.

\section{Ein Kulturzentrum als Spital}

In einem Kulturzentrum in Diyarbakir, das zu einem provisorischen Spital umfunktioniert worden war, treffen wir einige junge schwerverletzte Kämpfer, die meisten aus Kobanê. Viele ihrer Verletzungen wurden mittels Fixateur externe behandelt. Diese Patientinnen und Patienten werden von medizinischem Personal in freiwilligen Einsätzen unentgeltlich versorgt. Einige der Verletzten sind querschnittsgelähmt und werden nie mehr so kämpfen können, wie sie es sofort wieder tun wollen. Eine junge Frau hat eine schwere Hirnverletzung und kann nicht mehr reden. Sie zeigt Emotionen von Angst und dann Freude, wenn ihr das Victory-Zeichen gezeigt wird. Das ist das Zeichen ihrer Kampfgefährtinnen, da fühlt sie sich sicher. Trotz der schweren Verletzungen werden auch diese jungen Menschen wiederholt von der türkischen Polizei für erkennungsdienstliche Ermittlungen auf Polizeiposten abtransportiert. Was trotz aller Anstrengungen der medizinisch Unterstützenden in diesem provisorischen Spital dringend fehlt, ist ein professionelles und für die

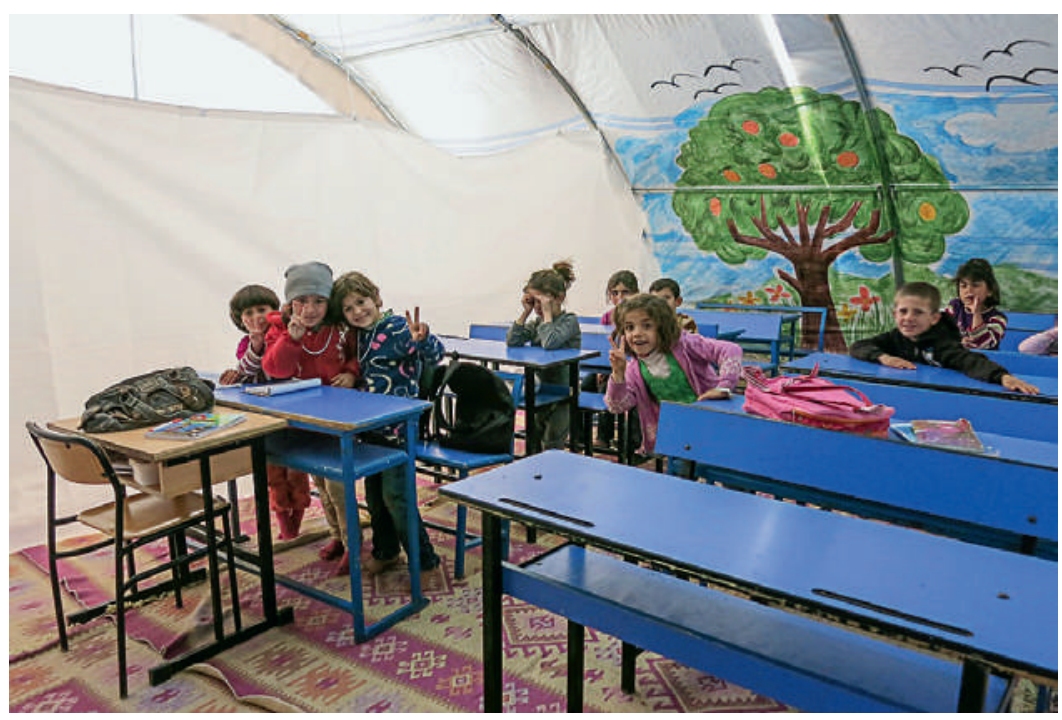

"Victory» - das Zeichen der Kämpfer - schon die Kinder kennen es, doch nicht alle schaffen es zu lächeln. spezielle Situation dieser Verletzten sensibilisiertes Rehabilitations-Team. Zwei bis drei Stunden wöchentlich kommt eine Physiotherapeutin freiwillig vorbei. Das ist viel zu wenig.

In der Nähe von Diyarbakir besuchen wir auch das von Kurden betreute Lager der Jesiden mit ca. 5000 Flüchtlingen. Wir treffen dort den ehemaligen kurdischen Präsidenten der türkischen Ärztegesellschaft aus Diyarbakir. Er begrüsst uns nur kurz, denn die Warteschlange der Patienten ist lang.

Am Abend vor unserer Rückreise, einer Reise, die für uns Schweizerinnen der Gruppe längst wie eine eigentliche Bildungsreise einer Volkshochschule geworden war, zeigt uns unser Gastgeber, ein Chirurg, die armenische Kirche in der Altstadt von Diyarbakir. Diese wurde erst vor drei Jahren mit armenischen Spende-

\section{Was dringend fehlt, ist ein professionelles} und für die spezielle Situation dieser Verletzten sensibilisiertes Rehabilitations-Team.

geldern wieder aufgebaut. Die Kirche ist heute ein Mahnmal für den armenischen Genozid, und für die Kurden ist sie auch ein Statement für ihre Multireligiosität und Multiethnizität.

Der Kriegsschauplatz im mittleren Osten, den wir mit einem Abklärungsauftrag für medico international Schweiz besuchten, ist nur einer von vielen Schauplätzen des Krieges in einer globalisierten Welt. Dass wir in so kurzer Zeit auf dieser Reise so viele Einblicke hatten, verdanken wir der kurdischschweizerischen Rheumatologin Birçan Turan und ihrer unermüdlichen Übersetzungs- und Informationsarbeit.

«Im neuen regionalen Machtkampf sind die kurdischen Akteure die einzige nennenswerte säkulare Kraft, die auch über ausreichend militärische Kapazitäten verfügt [...] eines muss allen Akteuren klar sein: Wenn Rojava in die Hände von IS fällt [...], ist dies nicht nur eine humanitäre Katastrophe [...], sondern auch eine entscheidende Wende im Machtkampf in der Region", schreibt der Kurdenkenner Thomas Schmidinger (Krieg und Revolution in Syrisch-Kurdistan, Mandelbaum Verlag, 2014).

Wir hoffen, dass das kleine zivile kurdische Projekt Rojava in der jetzigen politischen Umverteilung des Mittleren und Nahen Ostens nicht von globaleren Mächten und Interessen zermalmt wird.

Als Ärztinnen können wir überall und immer wieder unser humanitäres Engagement mobilisieren und in diesem Sinne bitten wir um Unterstützung des Nothilfe-Projektes von medico international Schweiz für Kobanê/Rojava. 